\section{A Simple Medium for the Demonstration of Chlamydospores of Candida albicans}

WHILE investigating the formation of chlamydospores by different species of the fungus Candida, it was observed that human serum as a medium gave good results. Cultures of Candida albicans 10, Candida tropicales 10, Candida krussei 10, and Candida stellatoidea 10, Candida guillarmondi 1 , and Candida pseudotropicales 1, were examined by the following technique: 24-hr. growth in Sabouræud's dextrose agar medium was emulsified with 1 c.c. of sterile normal saline; then 1 c.c. of this emulsion was added to a test-tube containing 4 drops of normal human serum, and the tube was incubated at $37^{\circ} \mathrm{C}$. in a water-bath. After a lapse of 24,48 and $72 \mathrm{hr}$., cover-slip preparations were examined under the microscope. In each instance, where cultural and biochemical tests for a strain conformed to those for Candida albicans, chlamydospores were produced after a lapse of $24 \mathrm{hr}$. Chlamydospores, however, did not develop with other types. Thus the above findings show that this method is very simple, loss expensive and less time-consuming than routine methods.

\section{R. Dutt-ChoudhuRi} RoBIN DUTT

Central Laboratory,

Chitaranjan Seva Sadan College of

Obstetrics, Gynæcology and Child Hoalth and

Chittaranjan Cancer Hospital, Calcutta.

\section{Growth of a Nematode in Tomato Plants grown on Sodium-deficient Water Cultures}

Host plants deficient in nitrogen, phosphorus, potassium, magnesium, sulphur and iron will support a population of the root-knot nematode Meloidogyne jaranica ${ }^{1}$, whereas plants on full nutrient and heavily infected with $M$. incognita acrita do not show deficioncies of these elements ${ }^{2}$. It has been reported ${ }^{3}$, however, that tomatoes infected with $M$. incognita acrita show less sodium in their tissues than do uninfected plants.

It may be that members of the genus Meloidogyne require large amounts of sodium during their development, and the following experiment was undertaken to test the effect of 'sodium deficiency' on the growth and reproduction of $M$. javanica grown in tomatoes. The terms 'sodium deficient' and 'sodium free' used here refer to plants grown in water cultures in which the presence of sodium has been reduced to a minimum.

All seeds used came from a single susceptible tomato plant of the variety Pan America. They were washed six times in water distilled from a silica still which was changed hourly. This water, which was used throughout the experiment, contained 0.0002 p.p.m. sodium.

Nutrient salts were re-crystallized as many as six times, and their sodium content estimated before use. The seeds were germinated on nylon mesh over distilled water. When the radicles were about $1 \mathrm{~cm}$. in length, a culture solution of half concentra. tion containing 0.0042 p.p.m. sodium was substituted for the distilled water. The concentrations of salts used and the methods of growth in polythene con. tainers have been described ${ }^{4}$.
Sodium was estimated, using a 'Si-Ro-Spec' atomic absorption instrument ${ }^{5}$, on a dry-weight basis. The methods used in infecting the plants ard in measuring the growth of the nematodes have been described ${ }^{1}$, except that the agar was dialysed against distilled water as a 5 per cent gel for five days with six changes before it was used. A 0.5 per cent solution of the dialysed agar was used as the infecting medium.

Infective larvæ of $M$. jaranica were washed several times over a sintered glass filter of pore diameter $5-15 \mu$ before being placed in the agar. The plants were exposed to infection for a period of $48 \mathrm{hr}$. and were then removed. The roots were washed in distilled water before being returned to the nutrients.

The plants were grown in a cooled glass-house from which dust contamination was reduced by a positive pressure of filtered air. A diurnal temperature variation between $60^{\circ}$ and $110^{\circ} \mathrm{F}$. was maintained during the experiment.

Plants grown in the presence of sodium were grown in culture solutions identical with those without sodium except that they had $0.1 \mathrm{~m}$.equiv./litre of sodium sulphate added. The roots and leaves of these plants were hydrolysed separately in silica Kjeldahl tubes using a mixture consisting of $5 \mathrm{ml}$. concentrated nitric acid, $1 \mathrm{ml}$. concentrated sulphuric acid and $1 \mathrm{ml}$. concentrated perchloric acid, all these chemicals being A.R. grade. Blanks consisting of these chemicals were included in all hydrolyses and sodium estimations.

Throughout the experiment the growth of plants in all treatments was similar. Plants were harvested at $7,14,21$ and 28 days, and their dry weights were similar, whether or not they were infected or whether or not they had sodium. At the final harvest, average weights of twelve plants from each of the two treat. ments were approximately the same $(6 \cdot 4 \mathrm{gm}$.$) .$

The amounts of sodium in the roots and leaves of these plants showed marked differences. Tomato plants grown on a 'sodium free' culture solution showed uniform amounts of sodium which ranged from 20 to 50 p.p.m. throughout the entire experiment for both roots and leaves; the roots always contained sodium in a slightly higher concentration than the leaves.

Plants grown with sodium showed a steady increase in uptake of this element in the roots throughout the entire experiment, ranging from 120 p.p.m. at the first (seventh day) harvest to 1,410 p.p.m. at the final (twenty-eighth day) harvest. The leaves of these plants, however, did not show an increase in sodium concentration throughout the experiment.

These results suggest that sodium accumulates in the roots of tomato plants when the amounts mentioned above of sodium are supplied. Infection was not consistent using agar, and although great care was taken to make this procedure as uniform as possible, in several instances plants remained uninfected.

Infected 'sodium-free' plants were harvested at 7,14 and 28 days, and galled roots were cut away from the plant and fixed in buffered 1 per cent osmium tetroxide at $p H \mathbf{H} \cdot 2$. They were washed in several changes of distilled water and at each harvest, fifty nematodes were dissected at random and their cross. sectional area measured as previously described ${ }^{1}$.

Growth of these nematodes on tomatoes containing 20-50 p.p.m. sodium was quite normal. They moulted, produced egg sacs, eggs and viable larva which were capable of re-infecting tomato seedlings, 